\title{
Dimer-Quadrupolar Quantum Phase Transition in the Quasi-One-Dimensional Heisenberg Model with Biquadratic Interaction
}

\author{
Kenji Harada, ${ }^{1}$ Naoki Kawashima, ${ }^{2}$ and Matthias Troyer $^{3}$ \\ 1 Department of Applied Analysis and Complex Dynamical Systems, \\ Graduate School of Informatics, Kyoto University, Kyoto 606-8501, Japan \\ ${ }^{2}$ Institute for Solid State Physics, University of Tokyo, Kashiwa 5-1-5, Chiba 277-8581, Japan \\ 3 Theoretische Physik, Eidgenössische Technische Hochschule Zurich, 8093 Zurich, Switzerland
}

(Dated: February 8, 2020)

\begin{abstract}
The quasi-one-dimensional $S=1$ Heisenberg antiferromagnet with a biquadratic term is investigated at zero temperature by quantum Monte Carlo simulation. As the magnitude of the inter-chain coupling is increased, the system undergoes a phase transition from a spontaneously dimerized phase to a Néel ordered or spin nematic phase. The numerical results suggest the possibility of an unconventional second-order transition in which the symmetry group characterizing one phase is not a subgroup of the other.
\end{abstract}

PACS numbers: $75.40 . \mathrm{Mg}, 75.10 . \mathrm{Jm}$

In the search for novel quantum phases and transitions, various spin models with interactions beyond the ordinary bilinear couplings have been investigated. Among such models, the $S=1$ Heisenberg model with biquadratic interaction is known to possess various interesting non-magnetic ground states, such as a dimerized and a Haldane phase in one dimension and a quadrupolar (or spin nematic) phase in two or more dimensions. In most magnetic systems the biquadratic term corresponds to a higher order exchange coupling and is much smaller in magnitude than the bilinear interactions, bearing no large effects upon the thermodynamic behavior. The investigation of this model at large values of the biquadratic term was thus mostly of academic interest. Recently, however, experiments [1] on optically trapped ultra-cold atoms opened new possibilities of flexible and controllable quantum systems. In particular, cold atoms in a periodic potential (e.g. an optical lattice), exhibit a superfluid to Mott-insulating phase transition [2], which was subsequently studied by quantum Monte Carlo simulation [3, 4, 5, [6] showing a potential capability of realizing various strongly-correlated quantum systems. More closely related to the present study, it was shown [7, [8] that $\mathrm{Na}$ atoms trapped in an optically generated periodic potential, forming a Mott insulator with one atom per lattice site, the hyper-fine $S=1$ spins in the insulating phase may be described as an $S=1$ Heisenberg antiferromagnet with a large biquadratic term, comparable in magnitude to the bilinear term.

In this Letter we present the results of quantum Monte Carlo simulations of the two-dimensional model with strong spatial anisotropy. When the anisotropy is varied this model exhibits a quantum phase transition from a dimerized phase to a spin nematic phase or a Néel phase, as shown in Fig. 1] The dimerized phase present in one dimension does not survive in the isotropic two- and three-dimensional model as conjectured by reference [7], but is stable only in anisotropic quasi-one-dimensional systems. Of particular interest is the quantum phase transition between the dimerized and the spin nematic

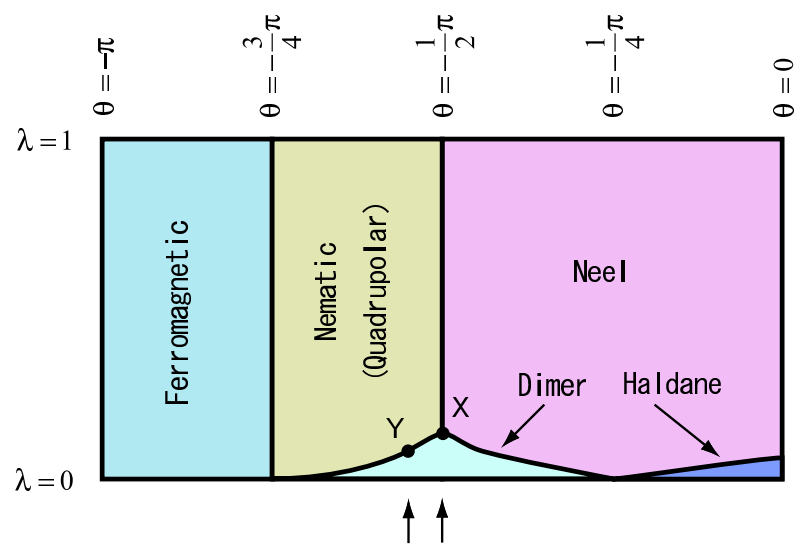

FIG. 1: A schematic phase diagram of the $S=1$ Heisenberg antiferromagnet with a biquadratic term. Two arrows indicate values of $\theta$ (see text for the definition) at which Monte Carlo simulations are carried out.

phase, at which we find that the correlation length is either infinite or at least very large $(\xi \gg 100)$. If the transition is of the second order, it must fall into an unconventional class of critical phenomena $9,10,11$, because the symmetry group characterizing one phase is not a sub-group of the other.

The model we consider is described by the following $S=1$ spin Hamiltonian:

$$
H=\sum_{\left(\boldsymbol{r}, \boldsymbol{r}^{\prime}\right)}\left(J_{\boldsymbol{r}, \boldsymbol{r}^{\prime}}^{(L)} \boldsymbol{S}(\boldsymbol{r}) \cdot \boldsymbol{S}\left(\boldsymbol{r}^{\prime}\right)+J_{\boldsymbol{r}, \boldsymbol{r}^{\prime}}^{(Q)}\left(\boldsymbol{S}(\boldsymbol{r}) \cdot \boldsymbol{S}\left(\boldsymbol{r}^{\prime}\right)\right)^{2}\right),
$$

where the couplings are parameterized by an angle parameter $\theta$ such that $J_{i j}^{(L)}=J_{i j} \cos \theta$, and $J_{i j}^{(Q)}=J_{i j} \sin \theta$. We focus on the region $\theta \leq 0$ and will specifically consider two cases $\theta / \pi=-1 / 2$ and -0.55 . While the model generally has the obvious $\mathrm{SU}(2)$ symmetry, it possesses an enlarged $\mathrm{SU}(3)$ symmetry in the case of $\theta=-\pi / 2$.

A rough sketch of the phase diagram was presented previously [12] and is reproduced in Fig. 1 For the onedimensional system, there are several special points at 
which exact results are available [13, 14, 15, 16], and the complete phase diagram has been established, except for the possibility of an intermediate phase between the ferromagnetic phase and the dimerized phase 17, 18, 19], whose presence or absence does not have any direct consequence to the present letter though [20]. In two dimensions the mean-field theory 21, 22] predicted the zerotemperature phase diagram as shown in the top part of Fig. 1] which was confirmed by Quantum Monte Carlo simulations 23, 24]. The main focus of the present Letter is the phase transition that occurs as we go from one dimension to two dimensions by increasing the coupling between chains. We introduce the anisotropy parameter $\lambda$ such that $J_{i j}=J$ when the bond $(i j)$ is along the chain $(x)$ to one direction and $J_{i j}=\lambda J$ if the bond $(i j)$ is perpendicular to the chains (the $y$ direction).

Our Monte Carlo simulations are based on the loop algorithm with non-binary loops 25]. The system sizes we explored range from $L_{x}=4$ to $L_{x}=192$ with a fixed aspect ratio $L_{x} / L_{y}=4$. For each data point, we typically perform a simulation of more than 800,000 Monte Carlo sweeps. The inverse temperature ranges from $\beta J=L_{x} / 2$ to $2 L_{x}$ for the system size $L_{x} \leq 128$. We have checked that the estimated values of quantities discussed here do not depend on the temperature once the inverse temperature exceeds $L_{x} / J$. Therefore, we consider that we can regard the estimates in the present letter as zero-temperature values. We have measured two observables: the quadrupolar moment and the dimerization parameter. While the former characterizes the breaking of the spin rotational symmetry, the latter characterizes the breaking of the translational symmetry. We define the quadrupolar moment as $Q \equiv\left(S^{x}\right)^{2}-\left(S^{y}\right)^{2}$. As dimerization order parameter, we use $D(\boldsymbol{r}) \equiv(-1)^{r_{x}}\left(H_{d}\left(\boldsymbol{r}+\boldsymbol{e}_{x}\right)-H_{d}(\boldsymbol{r})\right)$, where $H_{d}(\boldsymbol{r}) \equiv \operatorname{diag}\left(H_{\boldsymbol{r}, \boldsymbol{r}+\boldsymbol{e}_{x}}\right)$, and $\boldsymbol{e}_{x}$ is the lattice unit vector in the $x$-direction.

We first look at the special point $\theta=-\pi / 2$, which has an enlarged $\mathrm{SU}(3)$ symmetry. In this case, the quadrupolar moment can be transformed to the staggered magnetization by a 'staggered' SU(3) rotation. Because of the symmetry change exactly at $\theta=-\pi / 2$ and our previous numerical evidence in two dimensions, we conjecture that the phase boundary between the quadrupolar region and the Néel region is vertical and straight exactly at $\theta=-\pi / 2$ as depicted in Fig. [1and ends at the critical point (marked as ' $\mathrm{X}$ ' in Fig. 1). Along this line, both the quadrupolar moment and the staggered magnetization are finite. In order to locate the transition point, one usually computes the structure factors or, more conveniently, the Binder parameter (the dimensionless ratio of the moments of the order-parameter distribution function). However, they are integrated quantities and, in the present case, are dominated by short-range behavior of the correlation function. Therefore, we have used as our probe the correlation function between two distant points. In particular, the ratio of the two-point correlation function of the largest distance $\left(L_{x} / 2\right)$ to that of a

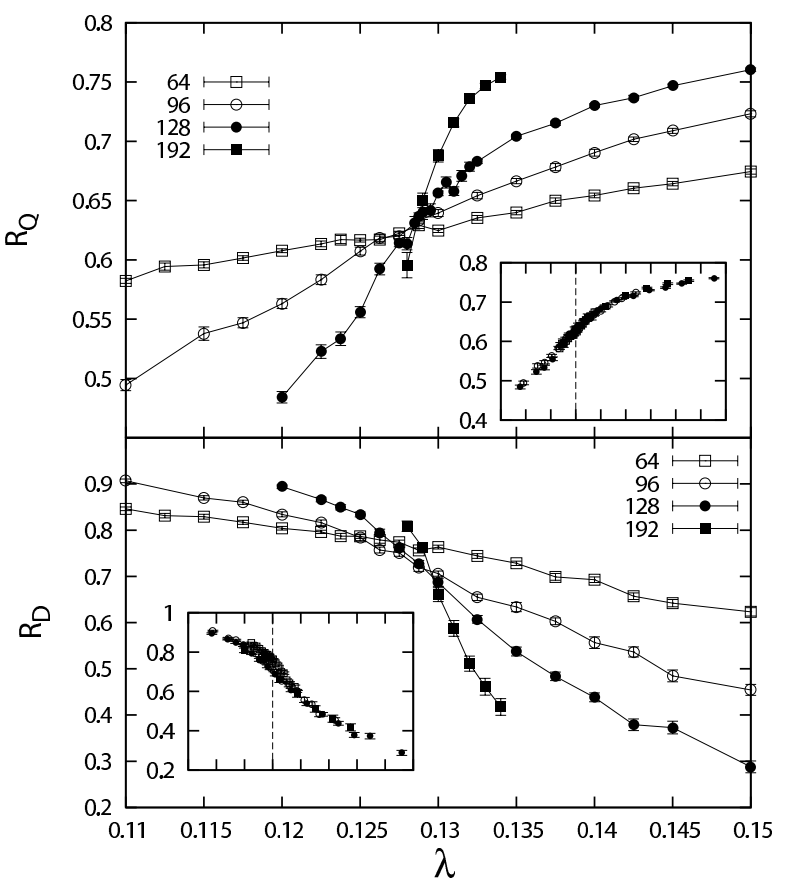

FIG. 2: The ratio of the two-point correlation function of the largest distance $\left(=L_{x} / 2\right)$ to that of a half of it $\left(=L_{x} / 4\right)$ for the quadrupolar moment (the upper panel) and the dimerization (the lower panel) at $\theta=-\pi / 2$. The insets are finite-sizescaling plots with $\lambda_{c}=0.1286, y_{\mathrm{Q}}=2.9$ (the upper panel) and $\lambda_{c}=0.1296, y_{\mathrm{D}}=2.54$ (the lower panel). The vertical dashed lines in the insets indicate the position of zero (the transition point).

half that distance $\left(L_{x} / 4\right)$ [26] is useful. For the quadrupolar moment, we compute $R_{Q}\left(L_{x}\right) \equiv G_{Q}\left(\frac{L_{x}}{2}\right) / G_{Q}\left(\frac{L_{x}}{4}\right)$ where $G_{Q}(l) \equiv\left\langle Q\left(l \boldsymbol{e}_{x}\right) Q(\mathbf{0})\right\rangle$. The correlation ratios for the dimerization $D$ are defined similarly. The correlation ratio for the quadrupolar moment is shown in the upper panel of Fig. 2] As expected, the curves for different system sizes share a common crossing point, and it is near $\lambda \sim 0.129$. A similar analysis is applied to the dimerization correlation function. (See the lower panel of Fig. 2]) Again all the curves cross each other in the vicinity of a single point. The location of this common crossing point is $\lambda \sim 0.129$ and $R_{D} \sim 0.75$. For a more systematic analysis, we also apply finite-size-scaling as shown in the insets of Fig. 2 From the quadrupolar moment, we obtain $\lambda_{c}=0.1287(8), y_{\mathrm{Q}}=1 / \nu_{\mathrm{Q}}=2.9(2)$. The critical exponent, $\eta_{\mathrm{Q}}$, can be estimated from the size dependence of the correlation function $G_{Q}(L / 2)$ by assuming $G_{Q}(L / 2) \propto L^{-\left(D-2+\eta_{Q}\right)}(D \equiv d+z)$ at the critical point estimated above. The results is $z+\eta_{\mathrm{Q}}=1.2(1)$. It is tempting to compare this result with the prediction of the deconfinement critical-phenomena (DCP). 9, 10, 11], Although it is not totally clear whether such a comparison is appropriate in spite of the difference in the symmetry of the systems (the four-fold rotational symmetry in 9 and [10] and the two-fold symmetry in the present case), 
the present result for $\eta_{\mathrm{Q}}$ is certainly larger than the value of the universality class of the three-dimensional Heisenberg model, which is consistent with the prediction of the DCP. A similar analysis for the dimerization yields $\lambda_{c}=0.1288(8), y_{\mathrm{D}}=2.5(2)$. As for $\eta_{\mathrm{D}}$, we do not quote an estimate here because a power law behavior has not been observed for a large enough range of the system size to establish a reliable estimate.

It should be kept in mind here, however, that these analyses are based on the assumption of a conventional critical point, which may not be justified in the present case. In fact, there are reasons to expect poor quality of the finite-size-scaling plot, if the present critical phenomenon is similar to the one discussed in Refs. [9, 10, 11]. Firstly, there must be two length scales (such as $\xi$ and $\xi_{\mathrm{VBS}}$ in 11]) diverging with different exponents. Therefore a good scaling plot is possible only for system sizes greater than the shorter (but still divergent) scale. Secondly, due to the existence of the secondary fixed point (analogous to the $\mathrm{U}(1)$ spin liquid fixed point in [11]) for the renormalization flow to the VBS fixed point, the apparent value of the scaling exponent $y$ in one phase can differ from that in the other, which could be fatal for an ordinary finite-size-scaling analysis.

We next look at the case $\theta=-0.55 \pi$ and examine the nature of the transition at the point ' $\mathrm{Y}$ ' in Fig. 11 In the previous case of $\theta=-\pi / 2$, if we regard the ground state as the Néel state rather than the spin nematic state, the symmetry group of the magnetic phase is a sub-group of that of the dimerized state. Therefore, the transition is not 'unconventional' even if it is of the second order. This is in contrast to the case studied previously [10, 11] where the lattice rotation (by 90 degrees) is spontaneously broken in dimerized phase whereas it is not broken in the Néel state. At $\theta=-0.55 \pi$, the $\mathrm{SU}(3)$ invariance does not exist and the model has only the natural SU(2) invariance. In the spin nematic phase, the symmetry related to the spin space is reduced to $Z_{2}$ due to the spontaneous quadrupolar moment whereas the spatial symmetry is the group of all the lattice translations. In the dimerized phase, on the other hand, the full SU(2) symmetry exists in the spin space whereas the spatial symmetry is reduced to the sub-group that does not contain odd translations in the $x$-direction. Therefore, in the conventional picture of second-order transitions, the transition between two such phases must be of the first order.

Recently, however, the possibility of second-order transitions was discussed extensively and a new paradigm, namely the deconfinement critical phenomenon, was proposed. 9, 10, 11], In this scenario, the VBS state is viewed as a state in which $S=1 / 2$ quasi-particles are confined by the potential that is sustained by the spontaneous VBS order to form $S=1$ elementary excitations. As the quantum phase transition is approached, the VBS order is weakened and so is the confinement potential. Eventually, the quasi-particles are deconfined at the critical point, giving rise to quasi-long-range order. It was also predicted that the two phases with apparently unre-

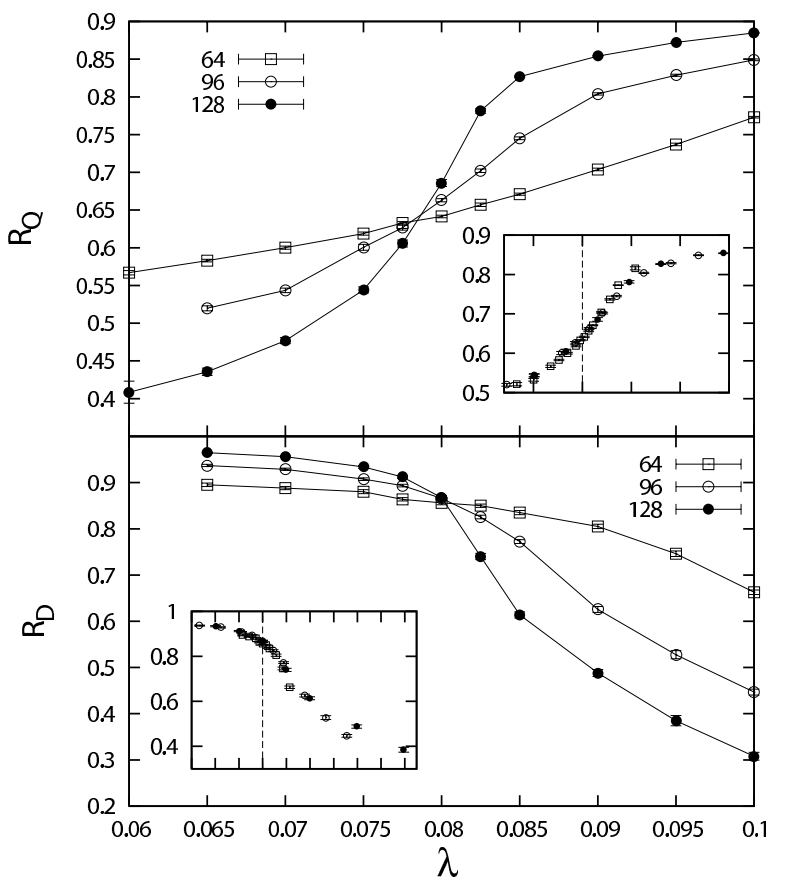

FIG. 3: Similar to Fig. 2 but for $\theta=-0.55 \pi$. The parameters used for the insets are $\lambda_{c}=0.0788, y_{\mathrm{Q}}=2.9$ (the upper panel) and $\lambda_{c}=0.0800, y_{\mathrm{D}}=2.8$ (the lower panel).

lated symmetries may have a direct second-order transition due to this mechanism. For this hypothetical class of phase transitions, there are efforts to find an example that exhibits such a transition [27]. However, so far no phase transition of any model is confirmed beyond reasonable doubt to match the theoretical description of DCP. In particular, it was shown in Ref. 28] that the DCP may not generically exist in models with U(1) symmetry.

In Fig. 3 we plot the correlation ratio at $\theta=-0.55 \pi$, We can see that both sets of curves cross near $\lambda=0.078$, an indication of a single phase transition between two phases. For the quadrupolar moment, the finite-sizescaling analysis yields $\lambda_{c}=0.079(1), y_{\mathrm{Q}}=2.8(2)$. From the size dependence of the correlation, we obtain $z+\eta_{\mathrm{Q}}=1.25(20)$. For the dimerization, on the other hand, we obtain $\lambda_{c}=0.080(1), y_{\mathrm{D}}=2.8(2)$. The system size dependence of the correlation ratio is not in perfect accordance with a first-order transition: if the transition is of the first order, the correlation ratios for both the quantities must be 1 simultaneously at the transition point. However, the ratio at the transition is almost constant and shows no visible trend towards unity. The correlation function decreases algebraically at the transition at least up to a distance of 128 lattice spacings.

Finally, in Fig. 4 we plot the energy per site as a function of $\lambda$. The system size dependence of the energy per site is small and no significant structure visible in this figure, in particular near the transition point $\lambda \sim$ 


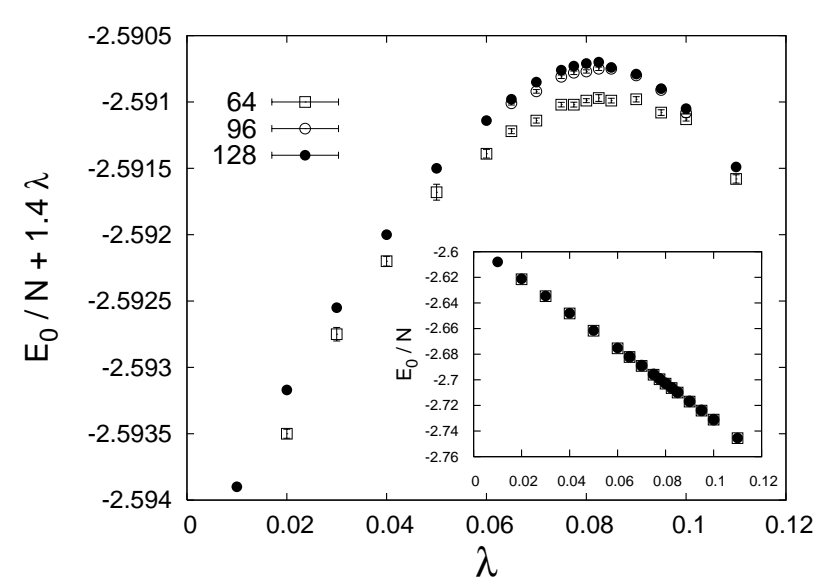

FIG. 4: The ground-state energy per site for $\theta=-0.55 \pi$, after subtraction of the dominant linear contribution $1.4 \lambda$, highlighting the absence of any cusp in the energy near the transition point $(\lambda \sim 0.079)$. The inset shows the raw data before subtraction.

0.079. It is still possible that the present transition is a very weak first-order transition of which the jump in the first derivative is impossible to observe within the present computational accuracy. Another open possibility is that the present phase transition is actually two second-order transitions very close to each other. While we cannot completely rule out these possibilities, we have obtained no evidence that positively supports either possibility.
The last and the most interesting scenario consistent with the present numerical evidences is a single secondorder transition. If this is the case, the transition is unconventional as discussed above, and may correspond to the predicted DCP scenario which is absent in generic $\mathrm{U}(1)$ symmetric models. 28] Because of the difference in the spatial symmetry of the model, namely, the lack of the lattice rotational symmetry in the present model, it is not clear how the phase transition studied in this Letter is related to the specific mechanism of deconfined criticality that involves monopole-type excitations. However, the generic scenario proposed in the previous studies $[9,10,11$, in which a secondary fixed point exists and the translational-invariance-breaking field becomes relevant at this point, may still apply to the present case. While the computations shown in this Letter are already fairly extensive (roughly $20 \mathrm{cpu} \cdot y e a r s)$, even larger-scale computations are being prepared for in order to single out the correct scenario for the transition in the $\mathrm{SU}(2)$ symmetric model and and to clarify its mechanisms.

We are grateful to F. Alet, A. Läuchli, S. Trebst and C. Wu for valuable and stimulating discussions. KH's work was supported by the 21st Century COE Program of Monkasho, Japan. MT acknowledges the Aspen Center for Physics. Preliminary studies were carried out on Beowulf clusters Asgard and Hreider at ETH Zürich. The main part of the computation was performed on SGI Altix3700/1280 at Supercomputer Center, Institute of Solid State Physics, University of Tokyo.
[1] S. Inouye, et al., Nature 392, 151 (1998).

[2] M. Greiner, et al., Nature 415, 39 (2002).

[3] G. G. Batrouni, et al., Phys. Rev. Lett. 89, 117203 (2002).

[4] V.A. Kashurnikov, N.V. Prokof'ev, and B V. Svistunov, Phys. Rev. A 66, 031601(R) (2002).

[5] S. Wessel, F. Alet, M. Troyer and G. G. Batrouni, Phys. Rev. A 70, 053615 (2004).

[6] S. Bergkvist, P. Henelius, and A. Rosengren, Phys. Rev. A 70, 053601 (2004).

[7] S. K. Yip, Phys. Rev. Lett. 90, 250402 (2003).

[8] A. Imambekov, M. Lukin and E. Demler, Phys. Rev. A 68, 063602 (2003).

[9] T. Senthil, A. Vishwanath, L. Balents, S. Sachdev, and M. P. A. Fisher, Science 303, 1490 (2004).

[10] T. Senthil, L. Balents, S. Sachdev, A. Vishwanath, and M. P. A. Fisher, Phys. Rev. B 70, 144407 (2004).

[11] T. Senthil, L. Balents, S. Sachdev, A. Vishwanath, and M. P. A. Fisher, J. Phys. Soc. Jpn. 74 Suppl., 1 (2005).

[12] K. Harada, N. Kawashima and M. Troyer, to appear in the proceedings of The 24th International Conference on Low Temperature Physics (LT24) (The AIP Conference Proceedings Series).

[13] B. Sutherland, Phys. Rev. B 12, 3795 (1975).

[14] L. A. Takhtajan, Phys. Lett. 87 A, 479 (1982).

[15] H. M. Babujian, Phys. Lett. 90 A, 479 (1982).

[16] A. Klümper, Euro. Phys. Lett. 9, 815 (1989).
[17] N. Kawashima, Prog. Theor. Phys. (Suppl.) 145, 138 (2002).

[18] A. V. Chubukov, Phys. Rev. B 43, 3337 (1991).

[19] G. Fáth and J. Sólyom, Phys. Rev. B 51, 3620 (1995).

[20] In our simulations, we observe an apparent transition point (between $\theta=-0.75 \pi$ and $\theta=-0.5 \pi$ ) at which the spontaneous dimerization seems to vanish. However, this apparent transition point is systematically closer to $\theta=$ $-0.75 \pi$ for larger systems, consistent with the absence of the intermediate phase and an exponentially-diverging correlation length at $\theta=-0.75 \pi$.

[21] H. H. Chen and P. M. Levy, Phys. Rev. B 7, 4267 (1973).

[22] N. Papanicolaou, Phys. Lett. A 116, 89 (1986); Nucl. Phys. B305[FS23], 367 (1988).

[23] K. Harada and N. Kawashima, J. Phys. Soc. Jpn. 70, 13 (2001).

[24] K. Harada and N. Kawashima, Phys. Rev. B 65, 52403 (2002).

[25] N. Kawashima and K. Harada, J. Phys. Soc. Jpn. 73, 1379 (2004).

[26] Y. Tomita and Y. Okabe, Phys. Rev. B 66, 180401(R) (2002).

[27] A. W. Sandvik and R. G. Melko, arXiv cond-mat/0604451

[28] A. B. Kuklov, N. V. Prokof'ev, B. V. Svistunov and M. Troyer, arXiv cond-mat/0602466 